\title{
REVISIÓN DE LOS ANTECEDENTES EN TRADUCCIÓN FINANCIERA: VISIÓN GENERAL DEL PANORAMA INVESTIGADOR EN ESTA ÁREA DE ESPECIALIZACIÓN
}

\author{
Elena Alcalde Peñalver*
}

\begin{abstract}
Resumen: En este artículo pretendemos realizar un recorrido sobre los principales trabajos publicados en relación a la traducción financiera, como muestra del panorama investigador que existe en la actualidad acerca de la misma. Para ello, describiremos en primer lugar nuestra propuesta clasificatoria de las distintas obras estudiadas y posteriormente las analizaremos dentro de su categoría correspondiente. El objetivo, tal y como señalaba Fink $(2005$, 3), es que esta revisión de los antecedentes sirva para identificar, evaluar y sintetizar la literatura existente y aportar una primera visión sobre los trabajos realizados hasta la época que tratan de la traducción financiera, un ámbito que no ha recibido hasta la fecha la atención de otras áreas de especialidad en traducción, como es por ejemplo el caso de la jurídica.
\end{abstract}

Palabras clave: Traducción financiera. Revisión antecedentes. Clasificación.

\footnotetext{
* Doctora en Traducción por la Universidad de Granada. Máster en Traducción e Interpretación por la Universidad de Granada. Licenciatura en Traducción e Interpretación por la Universidad de Granada en la combinación lingüística inglés-francés-español. BA (Honors) en Lenguas Modernas por la Universidad de Northumbria (Newcastle, United Kingdom) y Maîtrise en Lenguas Extranjeras (Mention Très Bien) por la Université de Provence, Aix-Marseille I, France. Máster en Traducción e Interpretación por la Universidad de Granada. Desde 2011 trabaja como investigadora y docente en el Departamento de Traducción e Interpretación de la Universidad de Granada. Granada, España. E-mail: ealcalde@ugr.es
} 


\title{
LITERATURE REVIEW ON FINANCIAL TRANSLATION: A FIRST OVERVIEW OF THE RESEARCH LANDSCAPE IN THIS AREA OF SPECIALIZATION
}

\begin{abstract}
The aim of this article is to look at the main studies published on financial translation to show the research landscape in this area of specialization. For the literature review, a classification model will be applied depending on the research objectives of the publications. First of all, this classification will be explained and then different publications will be analyzed in accordance with each category of the classification. The aim of this article is, as Fink $(2005,3)$ stated, to identify, evaluate, and synthesize existing literature on the topic and provide a first overview of what has been published to date in financial translation, which is considered an under-researched area compared to other specializations in the field of translation.
\end{abstract}

Keywords: Financial translation. Literature review. Classification.

\section{Introducción}

Considerando la variedad de obras con objetivos de investigación distintos que encontramos en relación a nuestro objeto de estudio, hemos decidido adoptar una propuesta clasificatoria dependiendo de la función que puedan desempeñar. Por un lado, comenzaremos analizando las obras que versan acerca del discurso financiero como lenguaje de especialidad. Este apartado lo dividiremos a su vez en los estudios de la metáfora en el discurso financiero, anglicismos, siglas y acrónimos y referencias culturales. Finalmente, analizaremos las publicaciones referentes a investigación en traducción financiera en sí, así como las que traten la práctica profesional de la misma. 


\begin{tabular}{|c|c|}
\hline \multicolumn{2}{|c|}{$\begin{array}{l}\text { PROPUESTA CLASIFICATORIA DE REVISIÓN } \\
\text { DE ANTECEDENTES }\end{array}$} \\
\hline $\begin{array}{l}\text { Discurso financiero como } \\
\text { lenguaje de especialidad }\end{array}$ & $\begin{array}{l}\text { Metáforas } \\
\text { Anglicismos } \\
\text { Siglas y acrónimos } \\
\text { Referencias culturales }\end{array}$ \\
\hline Traducción financiera & $\begin{array}{l}\text { Estudios de investigación en traducci- } \\
\text { ón financiera } \\
\text { Práctica de la traducción financiera }\end{array}$ \\
\hline
\end{tabular}

Tabla 1. Propuesta clasificatoria de la revisión de antecedentes

\section{Discurso financiero como lenguaje de especialidad}

En primer lugar, en relación a los estudios publicados sobre el discurso financiero, hemos considerado de gran utilidad el de Alcaraz y Hughes (2008) por su propuesta clasificatoria del español de los negocios, en el que se enmarca el discurso financiero, del que señalan su dinamismo y dependencia de la lengua inglesa. Este trabajo seguía la línea de una investigación previa en el campo, ya que en su artículo de 2001 Alcaraz se detenía a analizar el registro financiero en inglés. Consideraba que este merecía un tratamiento aparte puesto que era completamente distinto del de la economía y el comercio. Este autor señala que, debido a la supremacía de los Estados Unidos en el ámbito económico y al grado de coloquialismo del inglés de este país, el registro financiero, a su vez marcado por los riesgos, la especulación y el juego, venía a contagiarse de estas características. A esto se añadía el dominio del léxico anglosajón, que contrasta con el vocabulario de base latina del lenguaje económico (2001).

Talaván (2011) señala igualmente que conviene distinguir entre el lenguaje de la economía y el de las finanzas puesto que el registro económico es más formal y académico debido a sus raíces latinas. Esto conlleva, como han indicado otros investigadores, 
menores problemas de traducción que en el caso de las finanzas, caracterizado por su alto componente coloquial. Talaván menciona también otro aspecto recurrente en este campo como es el de las fronteras difusas entre las finanzas y el derecho, lo que ejemplifica con el caso de los documentos de hipotecas o seguros.

Asimismo, Gallego (2013) estudia, mediante un análisis de corpus, las locuciones preposicionales de la lengua general en el ámbito económico-comercial, lo que ha sido hasta ahora poco estudiado en la literatura del discurso de este ámbito y analiza las distintas posibilidades de traducción de las mismas.

\subsection{Metáforas}

La tradición del uso de las metáforas tiene como una de sus principales bases el modelo cognitivo, por el que se considera que la economía es comparable con el organismo. Ya en 1890 Alfred Marshall afirmaba que el «el razonamiento económico debería basarse en métodos análogos a los cálculos físicos y el tono empleado en el mismo debería volverse progresivamente más biológico» (Henderson, 1982, 149). Hewings (1990, 252), por su parte, defiende el uso de las metáforas en el discurso financiero como medio para facilitar y aumentar su comprensión, pues se hace recurso a una figura que representa una realidad hipotética como ejemplo de lo que realmente ocurre en este campo. Se trata de un medio a partir del cual el economista o experto en la materia es capaz de expresar nuevos conceptos que surgen en un campo de gran dinamismo como es el caso de las finanzas.

En relación a análisis comparativos español-inglés, encontramos el de Orts y Rojo (2009) que realizaron un estudio de corpus con textos de El Economista y The Economist. Partían de la hipótesis de que antes de que comenzara a desarrollarse la crisis económica, el número de metáforas utilizado en sentido positivo era mayor en comparación con las utilizadas en sentido negativo, mientras que después de la crisis la tendencia se invirtió. Esta hipótesis se confirmó tras 
la realización del estudio y este puso de manifiesto que el lenguaje financiero español no es tan rico en metáforas como el inglés.

Por otro lado, Gallego (2012) llevó a cabo un estudio en el que se analizan colocaciones con el término crise en francés en un corpus especializado y se identifican las metáforas que aparecen. El análisis revelaba que estas metáforas pueden asociarse a conceptos entre los que se encuentran los alimentos, enfermedades o catástrofes y que existe una clara tendencia a la traducción literal para solucionar las dificultades que puedan plantear. Igualmente, Gil (2012) señala desde su experiencia profesional como traductor financiero la gran cantidad de metáforas existentes en el discurso financiero. Finalmente, Orts $(2013,8)$ indica que la variedad de metáforas del discurso financiero obliga al traductor a convertirse en un «artífice literario, capaz de diseccionar el juego metafórico planteado en la lengua origen de la manera más exitosa, hermosa, coherente, redonda, informativa e inspiradora posible: en la versión meta».

\subsection{Anglicismos}

Desde mediados del siglo XX el español es mucho más permeable a la inclusión de términos procedentes del inglés debido a la creciente globalización de la economía y la influencia de Estados Unidos a nivel mundial (Pizarro, 2010, 116). Estas circunstancias hacen que se generen nuevos conceptos con sus correspondientes términos en el lenguaje económico inglés que se exportan a diferentes lenguas. Esto conlleva que en numerosas ocasiones no solo se exporte el concepto sino también el término, adaptado o no a la lengua española.

Orts y Almela (2009) afirman que el prestigio y el grado de especialización de los expertos ha impregnado el discurso económico en español de préstamos y adaptaciones de la lingua franca. En su estudio, distinguen entre neologismos de lujo y neologismos necesarios. Los primeros se incorporan por razones de prestigio, 
como es el caso de cash flow, hedge fund o call money, todos con un equivalente aceptado en español (flujo de caja, fondo de inversión libre y dividendo pasivo, respectivamente). Sin embargo, los expertos consideran el término en inglés más técnico o influyente. En el caso de los neologismos necesarios, el correspondiente en español no se encuentra tan ampliamente aceptado, como es el caso de joint venture o dumping (en estos casos, la terminología ha evolucionado y sí que encontramos un uso frecuente de los términos en español «combinación de negocios» $\mathrm{y}$ «competencia desleal» para joint venture y dumping, respectivamente). No obstante, es necesario señalar que debido al gran dinamismo del campo, lo que se traducía de una forma hace unos años puede evolucionar.

Por otro lado, Ribas $(1992,189)$ opina que los profesionales de los negocios se resisten a usar los equivalentes de los anglicismos por motivos más pragmáticos. De igual modo, Tapia (2000) señala que si la jerga económica es una de las más contaminadas por los extranjerismos, dentro de ella, la jerga financiera destaca por la profusión de términos y siglas incorporados directamente del inglés.

Ante esta situación, González (1995) aboga por una normalización terminológica en español ante el constante desarrollo de los mercados bancarios y bursátiles. Denuncia como uno de los problemas el que sean a menudo las empresas que venden los productos financieros las que los nombran, lo que resulta en una fuente de neologismos con el objetivo de buscar la originalidad y novedad para diferenciarse de la competencia. Con el fin de ilustrar esto, menciona el caso de una entidad bancaria española que presentaba como una gran primicia unos créditos «revolventes», para referirse a revolving en inglés, que en este caso tiene el sentido de autorrenovable.

Siguiendo esta misma línea, Russo (2002) resalta en mayor medida, por su propia experiencia como revisora y traductora del FMI, el elevado grado de dinamismo de la terminología financiera y su consiguiente dependencia del inglés, lo que ilustra con numerosos ejemplos. Sin embargo, señala que la adopción de anglicismos representa una solución legítima desde el punto de vista prác- 
tico y morfológico aunque en algunos casos resulte forzado desde el punto de vista semántico.

\subsection{Siglas y acrónimos}

Las siglas ocupan un lugar destacado en la terminología financiera debido a la importancia de diferentes organismos e instituciones, además de las denominaciones de distintos productos que, por economía lingüística, si están formados por varios términos, acaban convirtiéndose en siglas (caso por ejemplo de ETF para Exchange Traded Funds, que se utiliza con preferencia al término «fondos cotizados» en español).

En relación a la traducción de siglas, Pérez $(2003,622)$ afirma que el criterio recomendable en todos los libros de estilo es el de la traducción de los términos y de la reordenación de sus siglas, siempre y cuando tengan una traducción aceptada ampliamente en las diferentes lenguas de destino. Este es el caso por ejemplo del FMI.

Sin embargo, existen otras siglas que hacen referencia a organismos del ámbito anglosajón que no se traducen y se emplean en el orden en el que aparecen en la lengua de origen. A modo de ilustración podemos mencionar el índice LIBOR (London Interbank Offer Rate, Tipo de interés de oferta interbancario de Londres), el NASDAQ (National Association of Securities Dealers Automated Quotation, Sistema de cotización electrónica de la bolsa de los Estados Unidos). En estos casos se podría enunciar en un primer caso la traducción de las siglas en inglés para su contextualización y a continuación pasar a hacer uso únicamente de las siglas.

\subsection{Referencias culturales}

En relación a las referencias culturales y, debido a la cada vez mayor importancia de la globalización en el campo de la economía, ha existido una tendencia reciente hacia la estandarización 
terminológica, como lo ilustran las NIIF (Normas Internacionales de Información Financiera) en el campo de la contabilidad o los Incoterms en el ámbito del comercio internacional. Aunque esta tendencia globalizadora suponga la supresión de algunos problemas de traducción en las áreas económicas, algunas culturas siguen manteniendo sus singularidades (Alcaráz, 2007, 8). De esta forma, tal y como señala Engberg (2013) en relación al lenguaje de los textos jurídicos, los traductores financieros tendrán que tener en cuenta los aspectos lingüísticos de las expresiones y terminología que tengan relación con la cultura financiera del texto origen. Un ejemplo de esto lo constituyen las peculiaridades fiscales de cada país o la organización empresarial divergente, además de los nombres de organismos, ya que sería imposible traducir la estadounidense SEC (Securities and Exchange Commission) por la CNMV. Del mismo modo, Román (2010) pone como ejemplo las diferencias conceptuales entre el Mercado de futuros de Estados Unidos (Commodity Futures Trading Commission, CFTC) y el MEFF, cada uno con sus propias leyes reguladoras, lo que habrá que tener en cuenta en la traducción de documentos referidos a estos organismos. Por lo tanto, y aunque en teoría las funciones de regulación de los mercados de valores cumplan un mismo objetivo, se trata de realidades distintas, con diversos niveles de autonomía, competencias, etc.

Estos problemas se palian con la competencia temática del traductor, que deberá en su caso aportar las respectivas explicaciones necesarias para encontrar una solución ante estas diferencias. En este mismo sentido, Robin Bonthrone, traductor financiero de gran renombre en la combinación lingüística alemán-inglés, señala que con el intento de estandarización de la terminología financiera, que supone la implantación de las NIIF a nivel europeo, el hecho de contar con una mayor facilidad para traducir la terminología que aparezca en documentos financieros tendrá que compensarse con un mayor nivel de especialidad por parte del traductor para aportar un valor añadido al trabajo que ofertan (Balch, 2001). 


\section{Traducción financiera}

En primer lugar, es necesario señalar que no son numerosos los estudios de investigación referidos a la traducción financiera propiamente dicha y muchos menos los referidos a la enseñanza-aprendizaje de la traducción en el mencionado ámbito de especialidad. Respecto a los estudios referidos a la traducción en sentido estricto, los autores han dedicado un mayor esfuerzo investigador a la traducción jurídica y jurada (Román, 2008). Sin embargo, sí que se han publicado en los últimos años numerosos artículos de traductores financieros que reflexionan acerca de su propia experiencia, lo que nos permite a su vez acercarnos más a la realidad del mercado. Por lo tanto, dividiremos este apartado en publicaciones sobre la investigación en traducción financiera y sobre su práctica.

\section{Estudios de investigación en traducción financiera}

En relación a estudios que se limiten a la traducción financiera desde una perspectiva investigadora encontramos en primer lugar el de Pizarro (1998), que realiza un trabajo de investigación en derecho comparado en lo que a la legislación sobre la elaboración de informes financieros en el Reino Unido y España se refiere. La autora analiza las principales diferencias y semejanzas en el formato entre un país y otro y los problemas más frecuentes que se plantean a los traductores, que residen sobre todo en la terminología. Del mismo modo, señala en un trabajo posterior (Pizarro, 2001) que existe un lenguaje específico de los informes financieros que se enmarcaría dentro del registro económico y de entre cuyas características se podrían destacar el empleo de la voz pasiva sobre la activa, el empleo de tiempos verbales en presente o el mayor uso de grupos nominales múltiples sobre los simples.

Por otro lado, Mourier (2004) realiza un estudio de corpus en el que compila estados financieros en inglés y danés de empresas que cotizan en la bolsa de Copenhague. Los resultados de su es- 
tudio revelan que las traducciones de estos documentos son en su mayoría de poca calidad, lo que achaca a la falta de conocimiento temático en el área, tal y como había demostrado en un estudio anterior (1996).

Por otro lado, encontramos el estudio de Herrero (2011) que analiza a partir de una serie de criterios si se puede hablar de un registro financiero y un género financiero de forma independiente del económico. Hemos considerado su estudio como un punto de partida para nuestra investigación, ya que estimamos de gran utilidad adoptar la metodología empleada en su artículo para definir con mayor detalle el concepto de traducción financiera, lo que realizamos de forma exhaustiva en un trabajo anterior (Alcalde, 2014).

\subsection{Práctica de la traducción financiera}

En cuanto a los estudios dedicados a la reflexión sobre la práctica de la traducción financiera partiendo de la experiencia propia de estos profesionales, encontramos en primer lugar el de Durban (1996) que menciona la necesidad de adaptar y reescribir los textos de origen, siempre y cuando esté de acuerdo el cliente. Para ello menciona ejemplos de traducción de unos estados financieros del francés al inglés, como en el que afirma que si en francés se habla de una fábrica nueva situada en Ecoully-les-Rouen, la versión en inglés podría contar simplemente con una traducción en la que se refiriera a la nueva fábrica situada en la región de Normandía.

Por otro lado, Rynne (2001) reflexiona en su artículo sobre la traducción de los estados financieros y señala que no se trata de una actividad fácil debido a las repercusiones que estos documentos pueden tener para una empresa, por lo que defiende la necesidad de que los traductores cuenten con conocimientos especializados en este ámbito.

Martin (2006) aborda en su artículo el tema del trabajo del traductor financiero en el campo de los delitos financieros, lo que conlleva la traducción de documentos relacionados con el blanqueo de 
dinero o la financiación de actividades terroristas. La autora señala que este campo demanda una gran especialización por parte del traductor y un constante estudio de la evolución de las finanzas para estar al tanto de los nuevos términos y conceptos que puedan surgir.

Finalmente, en relación a la traducción de conceptos relacionados con la contabilidad y que se utilizan en gran medida en la redacción de documentos financieros, Zeff $(2007,296)$ señala los problemas que pueden surgir cuando se procede a su traducción, ya que un empleo diferente en el país de destino impediría su comprensión. Orts $(2013,6)$ recomienda a los traductores financieros conocer el sentido de los conceptos clave de la jerga, ya que en ocasiones no bastará con su traducción, sino que habrá que explicar al receptor de la traducción su significado. En este sentido, señala que son tres las opciones traductológicas con las que cuenta un traductor financiero: la incorporación, el calco o la adaptación, lo que dependerá del tenor del texto meta (expertos con conocimientos de inglés, expertos sin conocimientos de este idioma o público lego).

\section{Conclusiones}

A través de este artículo hemos podido identificar las principales referencias que se encuentran en el panorama investigador sobre traducción financiera en relación, por un lado, al discurso financiero y, por otro, a la traducción financiera en sí. Para este análisis de revisión de antecedentes hemos propuesto una clasificación con distintas categorías para organizar las variadas publicadas analizadas. Esta revisión de los antecedentes nos ha permitido adquirir un amplio bagaje sobre la traducción financiera y conocer las líneas de investigación tratadas hasta ahora en esta especialidad de traducción, a la que, tal y como hemos señalado anteriormente, no se le ha dedicado el mismo esfuerzo investigador en comparación a otras especialidades de traducción como la traducción jurídica. Consideramos pues que se ha cumplido el objetivo anunciado anteriormente, puesto que, tal y como señalaba Fink $(2005,3)$, esta revisión de 
los antecedentes ha servido para identificar, evaluar y sintetizar la literatura existente y aportar una primera visión sobre los trabajos realizados hasta la época que tratan de la traducción financiera.

\section{References}

Alcalde Peñalver, E. (2014). Caracterización y contextualización de la traducción financiera: estudio empírico-descriptivo de la situación académica y profesional en España. Tesis doctoral. Universidad de Granada.

Alcaraz Varó, E. (2001). Problemas metodológicos de la traducción del inglés de los negocios. En Proceedings. First International Conference on Specialized Translation. Barcelona: Universitat Pompeu Fabra. 11-21.

Alcaráz Varó, E. (2007). La sociedad del conocimiento, marco de las lenguas profesionales y académicas. En Alcaráz Varó, E. et al. (Eds.) Las lenguas profesionales y académicas. Madrid: Ariel. 3-12.

Alcaraz Varó, E. y Hughes, B. (2008). Diccionario de términos económicos, financieros y comerciales: inglés-español. Barcelona: Ariel.

Balch, T. (2001). Three days of the Balance Sheet, and Much More. ATA Chronicle, V. 30, 8. 13-14.

Durban, C. (1996). Meeting clients needs through specialization. ATA Chronicle, 25, 2. 12-13.

Engberg, J. (2013). Comparative Law for Translation: The Key to Successful Mediation between Legal Systems". En Legal Translation in Context. Professional Issues and Prospects. New Trends in Translation Studies, V. 4. 9-25. 
Fink, A. (2005) Conducting Research Literature Reviews: From the Internet to Paper. Thousand Oaks, California: Sage Publications.

Gallego Hernández, D. (2012). Traducción económica y corpus: del concepto a la concordancia. Aplicación al francés y al español. Alicante: Publicaciones Universidad de Alicante.

Gallego Hernández, D. (2013). Fraseología basada en corpus: un primer paso hacia un proyecto bilingüe (francés y español) especializado en el ámbito de la economía y los negocios. En Mogorrón Huerta, A. et al. (Eds.) Fraseología, opacidad y traducción. Frankfurt am Main: Peter Lang. 219-235.

Gil, J. (2012). Specialising in Financial Translation. eCPD Webinar. Recuperado el 14 de mayo de 2013 de http://www.ecpdwebinars.co.uk/specialising_in_ financial_translation.html

Henderson, W. (1982). Metaphor in economics. Economics. 18, 4. 147-157.

Herrero, L. (2011). La conveniencia de una clasificación de los documentos del ámbito financiero para la traducción especializada. Entreculturas, 3. 85-98.

Hewings, A. (1990). Aspects of the Language of Economics Textbooks. En Dudley-Evans A. y Henderson W. (Eds.). 29-42.

Martin, L. P. (2006). Financial Crimes. ATA Chronicle, 35, 5. 27-32.

Mourier, L. (1996). Translation challenges and quality problems in Inancial reporting”. En Grinsted, A. (Ed.) Language \& Business Life, 2. Kolding: Southern Denmark Business School.

Mourier, L. (2004). Communicating financial reporting across continents. Hermes, 32. 145-165.

Orts, M. A. (2013).Traduciendo una crisis. Guía para el camino. Revista Traditori, 5. 6-8. 
Orts, M. A. y Almela, A. (2009). Translating the Spanish economic discourse of the crisis: Dealing with the inevitability of English loanwords. IJES (International Journal of English Studies): Recent and Applied Corpus-Based Studies. 133-157.

Orts, M. A. y Rojo, A. M. (2009). Metaphor framing in Spanish economic discourse: a corpus-based approach to metaphor analysis in the Global Systemic Crisis. Comunicación presentada en la First International Conference of AELINCO (Corpus Linguistics Spanish Association), Universidad de Murcia. Recuperado el 5 de junio de 2011 de http://www.um.es/lacell/aelinco/contenido/pdf/13.pdf.

Pérez Berenguel, J. F. (2003). Glosario de errores comunes en la traducción económica y financiera». En Muñoz Martín, R. (Ed.) I AIETI. Actas del I Congreso Internacional de la Asociación Ibérica de Estudios de Traducción e Interpretación. Granada 12-14 de Febrero de 2003. Granada: AIETI. 619-628.

Pizarro Sánchez, I. (2001). El registro económico de la lengua inglesa: análisis del género 'memorias anuales' y de sus traducciones al inglés. Tesis doctoral. Universidad de Valladolid.

Pizarro Sánchez, I. (2010) Análisis y traducción del texto económico. La Coruña: Netbiblio.

Pizarro Sánchez, I. (1998). La traducción de informes financieros: problemas fundamentales. En Féliz Fernández, L. y Ortega Arjonilla E. (Eds.) II Estudios sobre Traducción e Interpretación, Tomo III. Actas de las II Jornadas Internacionales de Traducción e Interpretación de la Universidad de Málaga. Málaga: Centro de Ediciones de la Diputación de Málaga. 1009-1013.

Ribas Pujol, A. (1992). Las innovaciones financieras y su traducción al español. Edo Julià, M. (Ed.) Actes del I Congrès Internacional sobre Traducciò. Barcelona: Universitat Autònoma de Barcelona. 183-190.

Román Mínguez, V. (2008). La enseñanza de la traducción (inglés-español) de los tipos textuales del mundo de la empresa: aproximación metodológica y materiales. Tesis doctoral. Universidad de Málaga.

Román Mínguez, V. (2010). La información para accionistas e inversores de las sociedades del Ibex 35: una valiosa fuente documental para la enseñanza- 
aprendizaje de la traducción económico-financiera. Segundo Seminario Internacional, Seminario Permanente de Formación de Formadores en Traducción e Interpretación. Facultad de Traducción e Interpretación, Universidad de Granada.

Russo, A. (2002). Préstamos del inglés en la jerga económica: ¿es posible evitar el sobreendeudamiento lingüístico? En Hernúñez, P. y González, L. (Coords.) El español, lengua de traducción: Actas del I Congreso Internacional. Luxemburgo: Comisión Europea.

Rynne, J. (2001). Approaching the Translation of Spanish Financial Statements. The ATA Chronicle, 30, 6. 33-36.

Talaván, N. (2011). A University Handbook on Terminology and Specialized Translation. A Coruña: Netbiblo.

Tapia Granados, J. A. (2000). Sobre capital-riesgo y otros aspectos de la jerga económica. Puntoycoma, 64. Recuperado el 14 de mayo de 2013 de http:// ec.europa.eu/translation/bulletins/puntoycoma/64/pyc64.pdf.

Zeff, s. (2007). Some obstacles to global financial reporting comparability and convergence at a high level of quality. British Accounting Review, 39. 290-302.

Recebido em: 12/12/2014 Aceito em: 28/02/2015 